\title{
Miscibility and Inter-Polymer Interactions of the Poly(methyl acrylate)/Poly(vinyl acetate) Blend as Studied by NMR in Solution
}

\author{
K. TAKegoshi, Yoshimichi OHYA, and Kunio Hikichi* \\ Department of Polymer Science, Faculty of Science, \\ Hokkaido University, Sapporo 060, Japan
}

(Received July 10, 1992)

\begin{abstract}
To study the miscibility of poly(methyl acrylate)/poly(vinyl acetate) (PMA/ PVAc) blend, we observed ${ }^{1} \mathrm{H}$ NOE difference spectra. Inter-polymer NOEs are observed, indicating that PMA and PVAc are mixed on a molecular level. The intermolecular distance between the $\mathrm{CH}_{3}$ protons of PMA and $\mathrm{CH}$ proton of PVAc is estimated to be $3.4 \AA$ The temperature dependence of spin-lattice relaxation time of ${ }^{13} \mathrm{C}$ nuclei was examined to investigate the interpolymer interaction. The motion of polymer-chain is found more hindered in blend, indicating that there exists an inter-polymer interaction in solution.
\end{abstract}

KEY WORDS High-Resolution NMR / Polymer Blend / Poly(vinyl acetate) / Poly(methyl acrylate) / Inter-Polymer Interaction / Nuclear Overhauser Effect $/{ }^{13} \mathrm{C}$ Spin-Lattice Relaxation /

According to modern thermodynamical theories ${ }^{1}$ of polymer blends, the Gibbs free enegy of mixing is described by the following three contributions: (1) combinatorial entropy of mixing, (2) free volume difference between the component polymers, and (3) exchange interaction energy. For most polymer pairs, mixing is unfavorable because the combinatorial entropy of mixing is too small to overcome the disadvantage of free volume difference. A certain exothermic interaction should therefore be operative for dissimilar polymers to be miscible. For example, the inter-polymer hydrogen bonding brings about the miscibility of poly(vinyl alcohol)/poly(methacrylic acid) (PVA/PMAA) and PVA/poly(acrylic acid) (PVA/PAA). ${ }^{2}$ There are, however, a few miscible polymer paris, in which no specific interaction is operative. The free volume difference for these polymers is too small to compensate the combinatorial entropy of mixing. One such polymer blend is poly(methyl acrylate)/poly(vinyl acetate) (PMA/PVAc) blend. Nandi et al. applied the inverse gas chromatography method to PMA/PVAc in several solvents to examine thermodynamic interaction parameters, and concluded that the miscibility of PMA/PVAc is not due to a specific interaction. ${ }^{3}$

If there is a specific inter-polymer interaction between component polymers, molecular motion of the interacting region of individual polymer in the mixture is expected to be different from that of pure solution. Such a difference in molecular motion can be studied by observing ${ }^{13} \mathrm{C}$ spin-lattice relaxation time $T_{1}$. We have studied effects of blending on molecular motion in polycarbonate/poly(methyl methacrylate) (PC/PMMA) blend by ${ }^{13} \mathrm{C}-T_{1}$ experiments. ${ }^{4} T_{1}$-temperature curves of phenyl carbons of PC and methoxy carbon of PMMA in the mixture shift to high tem-

\footnotetext{
* To whom correspondence should be addressed.
} 
peratures as compared to those of pure polymers. The ${ }^{13} \mathrm{C}-T_{1}$ curves of other carbons are not influenced by mixing. These results indicate that ${ }^{13} \mathrm{C}-T_{1}$ is sensitive to polymer-polymer interaction in the mixture, and in a favorable case, one can determine the interacting region, such as the phenyl group of PC and the methoxy group of PMMA for PC/PMMA. In this work, we apply the ${ }^{13} \mathrm{C}-$ $T_{1}$ method to investigate whether there is any specific interaction between PMA and PVAc. We also study the miscibility on molecular level by observing the inter-polymer ${ }^{1} \mathrm{H}$-NOE.

\section{EXPERIMENTAL}

\section{Materials}

PMA and PVAc were obtained from ALDRICH Chemical Co., Inc. The weight average molecular weight/numer average molecular weight $\left(M_{w} / M_{n}\right)$ is $30700 / 10600$ for PMA and $124800 / 52700$ for PVPh. PMA and PVAc were dissolved in anhydrous acetone at a concentration of $\sim 5 \mathrm{wt} / \mathrm{v} \%$, and mixed for a day at an unit ratio of 5:5. Optically transparent films were obtained by casting the solutions on a glass plate at room temperature. The films were dried further under vaccum at $363 \mathrm{~K}$ for a week. The 5:5 film was dissolved again in deuterated acetone (acetone- $d_{6}$ ) at $35 \mathrm{wt} / \mathrm{v} \%$ for the ${ }^{1} \mathrm{H}$ NOE and ${ }^{13} \mathrm{C}-T_{1}$ experiments.

\section{NMR Measurements}

All NMR spectra were obtained on a JEOL JNM-GX270 spectrometer operating at 67.5 $\mathrm{MHz}$ for ${ }^{13} \mathrm{C}$ and $270 \mathrm{MHz}$ for ${ }^{1} \mathrm{H}$. The ${ }^{13} \mathrm{C}-$ $T_{1}$ experiments in acetone- $d_{6}$ solution at various temperatures were conducted by the conventional inversion recovery method. ${ }^{1} \mathrm{H}$ NOE difference spectra were obtained at $-30^{\circ} \mathrm{C}$ by the truncated driven NOE technique. ${ }^{5}$ The intensity of ${ }^{1} \mathrm{H}$ radio frequency irradiation was about $15 \mathrm{~Hz}$.

\section{RESULTS AND DISCUSSION}

\section{${ }^{1} \mathrm{H}$ NOE Experiments}

Figure 1 shows the ${ }^{1} \mathrm{H}$ NOE difference spectra at $-30^{\circ} \mathrm{C}$. The $\mathrm{CH}$ proton of PVAc at $4.88 \mathrm{ppm}$ was irradiated for $1 \mathrm{~s}$ (Figure 1a), and the $\mathrm{OCH}_{3}$ protons of PMA at $3.65 \mathrm{ppm}$ were irradiated for $1 \mathrm{~s}$ (Figure $1 \mathrm{~b}$ ). Both intrapolymer and inter-polymer NOEs are observed, which are all negative NOEs. It is found that the inter-polymer NOE can be observed only below $243 \mathrm{~K}$. At higher temperatures above $243 \mathrm{~K}$, the correlation time is presumably in a region where NOE is ineffective. These NOE results show that PMA and PVAc are mixed intimately on the molecular level.

The relative intensity of NOE is plotted against irradiation time. The intensity at an irradication time of $10 \mathrm{~s}$ was taken as $100 \%$. Becuase of spectral overlapping, we could not obtain accurate intensity except for those of

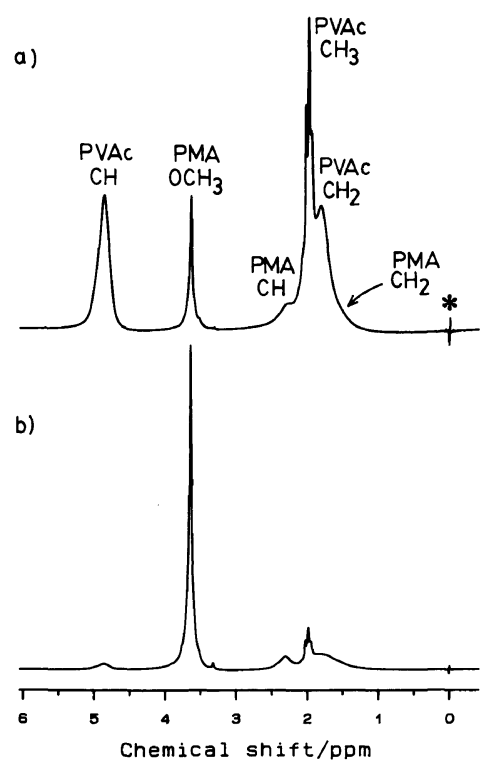

Figure 1. ${ }^{1} \mathrm{H}$ NOE difference spectra of PMA/PVAc $=$ $5: 5$ mixture in acetone $-d_{6}$ at $243 \mathrm{~K}$. (a) The $\mathrm{CH}$ proton of PVAc is irradiated for and (b) the $\mathrm{OCH}_{3}$ protons are irradiated for $1 \mathrm{~s}$. The dispersion-like peak marked with * is from tetramethylsilane added as an internal chemical shift reference. 


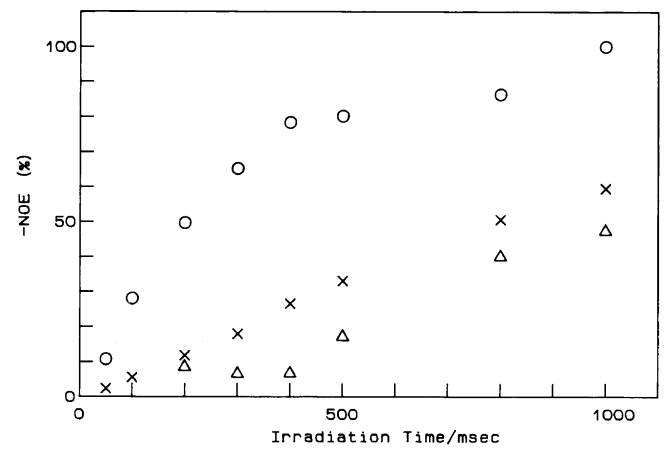

Figure 2. Irradiation time dependence of intensity of NOE difference spectra of PMA/PVAc $=5: 5$ mixture in acetone- $d_{6}$ at $243 \mathrm{~K}$. The $\mathrm{CH}$ proton of PVAc is irradiated. Marks $\mathrm{O}, \times$, and $\triangle$ denote the $\mathrm{CH}_{2}$ protons of PVAc, the $\mathrm{OCH}_{3}$ protons of PMA, and the $\mathrm{CH}$ proton of PMA, respectively.

the $\mathrm{OCH}_{3}$ and the $\mathrm{CH}$ protons of PMA and the $\mathrm{CH}$ proton of PVAc. Figure 2 shows the results of NOE experiments when the $\mathrm{CH}$ proton of PVAc is irradiated. As for the intra-polymer NOE, the intensity of the $\mathrm{CH}_{2}$ of PVAc was estimated from the overlapped signals at $1 \sim 3 \mathrm{ppm}$ by fitting them to a sum of four Lorentzian lines. Although the signal of the $\mathrm{CH}_{3}$ protons of PVAc is splitted due to tacticity, we simply adopted one Lorentzian line for it. The other three Lorentzian lines correspond to the $\mathrm{CH}_{2}$ protons of PVAc and $\mathrm{CH}$ and $\mathrm{CH}_{2}$ protons of PMA. As for interpolymer NOE, intensities of the $\mathrm{CH}$ and the $\mathrm{OCH}_{3}$ of PMA are plotted.

It is of interest to note that the initial build-up of the inter-polymer NOE observed for the main-chain $\mathrm{CH}$ of PMA is much slower than that of the side-chain $\mathrm{OCH}_{3}$ of PMA. This behavior suggests that the magnetization is first transferred to the side-chain of PMA by NOE and then to the main-chain of PMA by the spin diffusion. The proximity of the $\mathrm{CH}$ of PVAc and the $\mathrm{OCH}_{3}$ of PMA is, therefore, suggested, leading to inter-polymer hydrogen bonding. Such hydrogen bonding would change chemical shift of ${ }^{1} \mathrm{H}$ - and ${ }^{13} \mathrm{C}$ involved. No apparent chemical shift change by mixing is observed for ${ }^{13} \mathrm{C}$ and ${ }^{1} \mathrm{H}$ signals

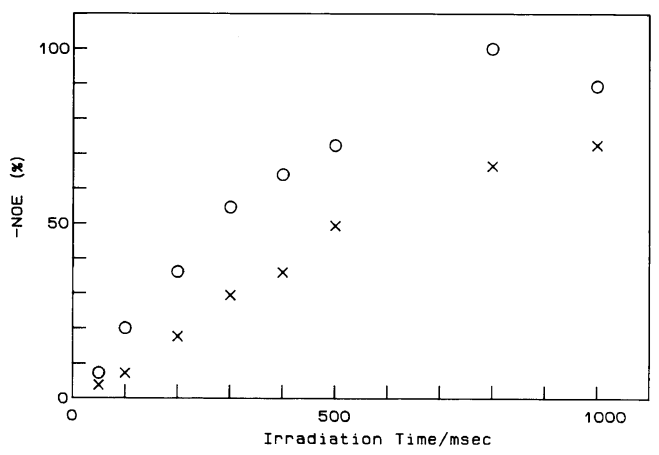

Figure 3. Irradiation time dependence of intensity of NOE difference spectra of PMA/PVAc $=5: 5$ mixture in acetone- $d_{6}$ at $243 \mathrm{~K}$. $\mathrm{OCH}_{3}$ protons of PMA are irradicated. Marks $O$ and $\times$ denote the $\mathrm{CH}$ proton of PMA and the $\mathrm{CH}$ proton of PVAc, respectively.

\section{at $223-298 \mathrm{~K}$.}

From the initial build-up rates of NOE in Figure 2, we evaluated an inter-polymer distance as follows. We assumed the standard bond lengths and angles for the main chain $\left(\mathrm{CH}-\mathrm{CH}_{2}\right)$ of PVAc. We also assumed that the $\mathrm{CH}$ and one of the $\mathrm{CH}_{2}$ protons take the anti conformation. An average intra-polymer distance between the $\mathrm{CH}$ and the two $\mathrm{CH}_{2}$ protons of PVAc is estimated to be $2.7 \AA$. The initial build-up rate of the $\mathrm{OCH}_{3}$ of PMA is $1 / 4$ of that of the $\mathrm{CH}_{2}$ of PVAc. Since the initial build-up rate is inversely proportional to the 6th power of the inter-proton distance, the inter-polymer distance between the $\mathrm{CH}$ proton of PVAc and $\mathrm{OCH}_{3}$ protons of PMA is evaluated to be $3.4 \AA$. The distance is short enough to cause NOE but probably too long to bring about chemical shift change.

In Figure 3, NOE intensities of the $\mathrm{CH}$ of PMA and the $\mathrm{CH}$ of PVAc when the $\mathrm{OCH}_{3}$ signal of PMA is irradiated are plotted. The behavior of inter-polymer NOE of the $\mathrm{CH}$ proton of PVAc is similar to that of the $\mathrm{OCH}_{3}$ protons of PMA in Figure 2. Other protons $\left(\mathrm{CH}_{3}\right.$ and $\left.\mathrm{PH}_{2}\right)$ of PVAc also show similar behavior.

\section{${ }^{13} \mathrm{C}-T_{1}$ Measurements}

Figures 4 and 5 show the observed tem- 


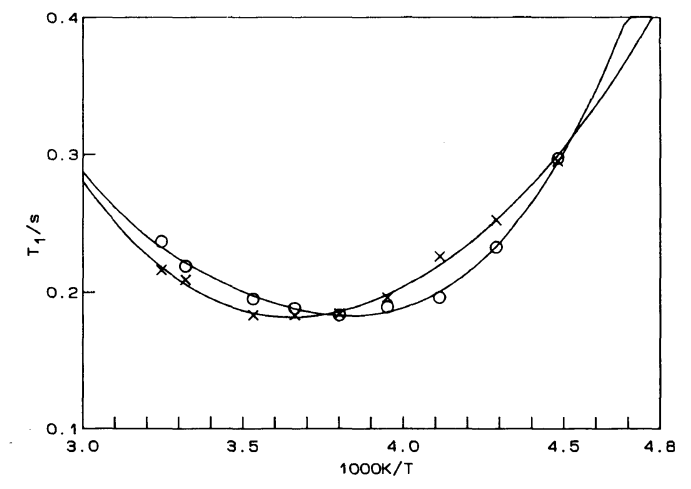

Figure 4. ${ }^{13} \mathrm{C}-T_{1}$ curves of the $\mathrm{CH}$ carbon of pure PMA $(O)$ and PMA/PVAc $=5: 5$ mixture $(x)$ in acetone$d_{6}$. The solid lines through the data points are "bestfitted" lines described by parameters listed in Table I.

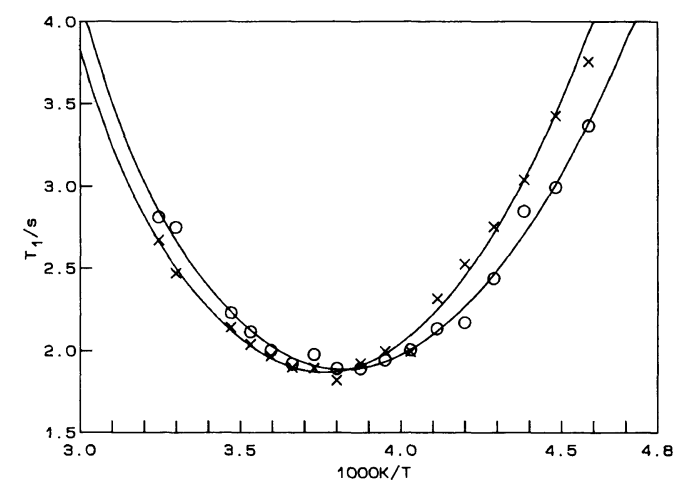

Figure 5. ${ }^{13} \mathrm{C}-T_{1}$ curves of the $\mathrm{OCH}_{3}$ carbon of pure PVAc $(O)$ and PMA/PVAc $=5: 5$ mixture $(x)$ in acetone$d_{6}$. The solid lines through the data points are "bestfitted" lines described by parameters listed in Table I.

perature dependence of ${ }^{13} \mathrm{C}-\mathrm{T}_{1}$ of the $\mathrm{CH}$ main-chain carbon of PMA (Figure 4) and that of the $\mathrm{C}=\mathrm{O}$ carbon of PVAc (Figure 5) in pure $(O)$ and mixed $(\times)$ states. The ${ }^{13} \mathrm{C}-T_{1}$ curve in he mixed state shifts toward higher temperatures, showing that mixing affects the molecular motion of PMA and PVAc. ${ }^{13} \mathrm{C}-T_{1}$ curves of other carbons also show the similar behavior.

To estimate the blending effects on molecular motion more quantitatively, we analyze the observed ${ }^{13} \mathrm{C}-T_{1}$. The conventional ${ }^{13} \mathrm{C}$ ${ }^{1} \mathrm{H}$ dipolar relaxation is written as ${ }^{6}$

$$
\begin{aligned}
T_{1}^{-1}= & A\left\{J\left(\omega_{\mathrm{C}}-\omega_{\mathrm{H}}\right)+3 J\left(\omega_{\mathrm{C}}\right)\right. \\
& \left.+6 J\left(\omega_{\mathrm{C}}+\omega_{\mathrm{H}}\right)\right\}
\end{aligned}
$$

where $A$ describes the strength of ${ }^{13} \mathrm{C}-{ }^{1} \mathrm{H}$ dipolar interaction and $J(\omega)$ is the spectral density function. $\omega_{\mathbf{H}}$ and $\omega_{\mathrm{C}}$ are the Larmor frequencies $\left(\mathrm{rad} \mathrm{s}^{-1}\right)$ of ${ }^{1} \mathrm{H}$ and ${ }^{13} \mathrm{C}$, respectively. For isotropic motion, $J(\omega)$ is the Lorentzian function as

$$
J(\omega)=\frac{2 \tau}{1+\omega^{2} \tau^{2}}
$$

where $\tau$ is the correlation time of isotropic motion. Since all observed ${ }^{13} \mathrm{C}-T_{1} \mathrm{~s}$ are not symmetric to the inverse of temperature, they are not well described by a simple isotropic motion (eq 2). The Cole-Cole distribution of a correlation time of motion ${ }^{7}$ was found insufficient to describe the observed $T_{1}$ curves. We adopted a conformational jump model, a model of motion of flexible-chain such as vinyl-polymer and polyether. ${ }^{8}$ The details of this model are found in ref 8 . In the conformational jump model, the specral density function $J(\omega)$ (eq 2 ) is given as

$$
\begin{aligned}
& J(\omega)=\frac{2 \theta \rho(\theta-\rho)}{(\theta-\rho)^{2}+\omega^{2} \theta^{2} \rho^{2}} \\
& \times\left\{\left(\frac{\theta}{2 \rho}\right)^{1 / 2}\left[\frac{\left(1+\omega^{2} \theta^{2}\right)^{1 / 2}+1}{1+\omega^{2} \theta^{2}}\right]^{1 / 2}\right. \\
& \left.+\left(\frac{\theta}{2 \rho}\right)^{1 / 2} \frac{\omega \theta \rho}{\theta-\rho}\left[\frac{\left(1+\omega^{2} \theta^{2}\right)^{1 / 2}-1}{1+\omega^{2} \theta^{2}}\right]^{1 / 2}-1\right\}
\end{aligned}
$$

where $\rho$ and $\theta$ denote the correlation time for conformational jump and that for isotropic mode of sub-chain motion, respectively. Although this model is postulated for the main-chain motion, we assume that it is valid for side-chain motion. It is because the ${ }^{13} \mathrm{C}-T_{1}$ of side-chain has a similar feature to those of main-chain, in particular, a very similar $T_{1}$ minimum temperature.

For least-squares fitting, we took the activiation energy $E_{\mathrm{a}}^{\mathrm{i}}$, correlation times at in- 
Table I. "Best-fitted" parameters to the ${ }^{13} \mathrm{C}-T_{1}$ data

\begin{tabular}{|c|c|c|c|c|}
\hline & $A / 10^{8} \mathrm{~s}^{-2}$ & $E_{\mathrm{a}} / \mathrm{kcal} \mathrm{mol}^{-1}$ & $\tau_{0} / 10^{-13} \mathrm{~s}$ & $\theta / \rho^{\mathrm{b}}$ \\
\hline \multicolumn{5}{|l|}{ PMA CH } \\
\hline Pure & $6.53 \pm 0.19$ & $3.8 \pm 0.1$ & $13.7 \pm 3.0$ & $0.02 \pm 0.04$ \\
\hline Mixture & $8.64 \pm 0.53$ & $4.8 \pm 0.6$ & $5.4 \pm 4.6$ & $1.7 \pm 1.1$ \\
\hline \multicolumn{5}{|l|}{$\mathrm{PMA} \mathrm{CH}_{3}$} \\
\hline Pure & $1.57 \pm 0.10$ & $3.4 \pm 0.3$ & $25.9 \pm 11.0$ & $0.02 \pm 0.01$ \\
\hline Mixture & $1.48 \pm 0.08$ & $3.7 \pm 0.2$ & $15.8 \pm 6.2$ & $0.002 \pm 0.02$ \\
\hline \multicolumn{5}{|l|}{$\mathrm{PMAC}=\mathrm{O}$} \\
\hline Pure & $0.74 \pm 0.04$ & $4.5 \pm 0.4$ & $4.6 \pm 3.0$ & $1.4 \pm 0.8$ \\
\hline Mixture & $0.75 \pm 0.04$ & $5.0 \pm 0.4$ & $2.4 \pm 1.6$ & $1.6 \pm 0.9$ \\
\hline \multicolumn{5}{|l|}{ PVAc $\mathrm{CH}_{3}$} \\
\hline Pure & $1.66 \pm 0.08$ & $4.5 \pm 0.2$ & $4.4 \pm 2.3$ & $0.3 \pm 0.3$ \\
\hline Mixture & $1.51 \pm 0.04$ & $4.7 \pm 0.2$ & $2.7 \pm 0.8$ & $0.05 \pm 0.04$ \\
\hline \multicolumn{5}{|c|}{ PVAc $C=O$} \\
\hline Pure & $0.79 \pm 0.02$ & $5.2 \pm 0.2$ & $1.4 \pm 0.5$ & $1.1 \pm 0.4$ \\
\hline Mixture & $0.77 \pm 0.02$ & $5.3 \pm 0.2$ & $1.3 \pm 0.5$ & $0.7 \pm 0.2$ \\
\hline
\end{tabular}

a Error is $\sigma$.

b See eq 3.

finite temperature $\tau_{0}^{\mathrm{i}}$ for conformational jump $(i=\rho)$ and isotropic mode of sub-chain $(i=\theta)$, and $A$ representing the strength of ${ }^{13} \mathrm{C}-{ }^{1} \mathrm{H}$ dipolar interaction. In the calculation, convergence of our least-squares fitting program based on the Gauss-Newton algorithm was very unstable because of a strong correlation between the two correlation times. To overcome this difficulty, we made the following assumption; the ratio of the two correlation times does not change significantly for the temperature range studied. If the activation energy of conformational jump does not differ significantly from that of isotropic mode of sub-chain, the ratio of the correlation times should not change drastically for the temperature range studied. The number of parameters was four, $A$, the ratio $\theta / \rho, E_{\mathrm{a}}$, and $\tau_{0}$ for isotropic mode of sub-chain. The solid lines in Figures 4 and 5 are the "bestfitted" lines with the parameters in Table I. The fitting is satisfactory, showing that assumption is good. ${ }^{13} \mathrm{C}-T_{1}$ curves for other carbons are also fitted and parameters are shown in Table I. We could not obtain $T_{1}$ data for the main-chain carbons of PVAc with sufficient accuracy amenable to the above analysis, because of line-splitting/broadening due to tacticity.

It is of interest to note that by mixing all activation energies of molecular motion of PMA/PVAc increase by $0.1-1 \mathrm{kcal} \mathrm{mol}^{-1}$. This tendency is also confirmed when we assume the Cole-Cole distribution of correlation time. Apparently, PMA and PVAc interact with each other in acetone. The increased activation energies in the mixture suggest that either the transition state of molecular motion is destabilized or the ground state is stabilized (or both). By taking into account the proximity of the side-chains of PMA and main-chain of PVAc found by the NOE experiments, the ground states of PMA and PVAc may be stabilized by mixing.

\section{CONCLUSION}

In contrast to PC/PMMA, in which only the ${ }^{13} \mathrm{C}-T_{1} \mathrm{~s}$ of the phenyl group of $\mathrm{PC}$ and the methoxy group of PMMA shift toward higher temperatures by mixing, all ${ }^{13} \mathrm{C}-T_{1}$ curves of PMA/PVAc shift toward higher temperatures. This probably suggests that there is non-specific interaction between PMA 
and PVAc. The inter-polymer NOE results do not, however, support this view. The NOE results suggest that there is a specific interaction between the $\mathrm{CH}$ group of PVAc and $\mathrm{OCH}_{3}$ group of PMA, probably hydrogen bonding interaction. The interaction is too weak to affect specifically the molecular motion of the groups involved. Although each hydrogen bonding is weak, the overall interaction on a whole polymer chain should become large to restrict large scale molecular motion. It is true that PMA and PVAc are very similar in their $P-V-T$ properties ${ }^{3}$ but the mixing is not like mixing two identical polymers.

Acknowledgments. This work was partly supported by a Grant-in-Aid for Scientific Research (No. 02554014) from the Ministry of
Education, Science, and Culture of Japan.

\section{REFERENCES}

1. (a) P. J. Flory, J. Am. Chem. Soc., 87, 1833 (1965);

(b) D. Patterson, Macromolecules, 2, 672 (1969).

2. X. Zhang, K. Takegoshi, and K. Hikichi, Polym. J., 23, 79 (1991).

3. A. K. Nandi, B. M. Mandal, and S. N. Bhattacharyya, Macromolecules, 18, 1454 (1985).

4. A. Asano, K. Takegoshi, and K. Hikichi, Polym. J., 24, 473 (1992).

5. G. Wagner and K. Wüthrich, J. Magn. Reson., 33, 675 (1979).

6. A. Abragam, "Principles of Nuclear Magnetism," Oxford, 1961.

7. F. Heatley and A. Begum, Polymer, 17, 399 (1976).

8. (a) B. Valueur, J.-P. Jarry, F. Geny, and L. Monnerie, J. Polym. Sci. Polym. Phys. Ed., 13, 667 (1975); (b) F. Geny and L. Monnerie, J. Polym. Sci., Polym. Phys. Ed., 17, 131, 141 (1979). 